\title{
Entomopathogenic fungi: unconventional applications
}

\author{
Anna Litwin $(\mathbb{D} \cdot$ Monika Nowak $\mathbb{D}$ - Sylwia Różalska $\mathbb{D}$
}

Published online: 10 February 2020

(C) The Author(s) 2020

\begin{abstract}
Entomopathogenic fungi (EPF) are microorganisms that cause fatal diseases of arthropods. The infection process involves several stages that consist of direct contact of the fungus with the surface of the cuticle of the attacked insect. The factors that determine the effectiveness of the infection process include lytic enzymes, secondary metabolites, and adhesins produced by EPF. Because of their high insecticidal effectiveness, these fungi are commonly used as biopesticides in organic farming. As the environment and farmlands are contaminated with many compounds of anthropogenic origin (e.g., pesticides), the effects of these toxic compounds on EPF and the mechanisms that affect their survival in such a toxic environment have been studied in recent years. This review presents information on the capacity of EPF to remove toxic contaminants, including alkylphenols, organotin compounds, synthetic estrogens, pesticides and hydrocarbons. Moreover, these
\end{abstract}

Electronic supplementary material The online version of this article (https://doi.org/10.1007/s11157-020-09525-1) contains supplementary material, which is available to authorized users.

A. Litwin · M. Nowak · S. Różalska $(\bowtie)$

Department of Industrial Microbiology and

Biotechnology, Institute of Microbiology, Biotechnology

and Immunology, Faculty of Biology and Environmental

Protection, University of Lodz, 12/16 Banacha Street,

90-237 Lodz, Poland

e-mail: sylwia.rozalska@biol.uni.lodz.pl fungi produce numerous secondary metabolites that can be potentially used in medicine or as antimicrobial agents. Despite their huge potential in biocontrol processes, the use of EPF has been underestimated due to a lack of knowledge on their abilities. In our work, we have presented the available data on the possibilities of the additional and unconventional use of these microorganisms.

Keywords Entomopathogenic fungi - Toxic compounds · Nanoparticle biosynthesis .

Biodegradation

\section{Introduction}

Entomopathogenic fungi (EPF) are bioinsecticides with an ability to infect and kill arthropods. Although they are mainly isolated from arthropod carcasses, their natural habitat is soil (Behie and Bidochka 2014). These fungi are categorized in six classes: Oomycetes, Chytridiomycota, Microsporidia, Entomophtoromycota, Basidiomycota, and the most common Ascomycota. The main function of EPF in the environment is the biocontrol of the insect population. The literature data provide information on the infectious properties of EPF, the infectious process (Skinner et al. 2014; Lacey et al. 2015; Mascarin and Jaronski 2016), and the use of these microorganisms in commercial 
preparations as biopesticides (Jaihan et al. 2016; RíosMoreno et al. 2016).

In the last few decades, the constantly increasing environmental pollution is a serious concern. Every day substances are being introduced in the environment that have an adverse effect on the balance of the ecosystem and living organisms. Fungi that inhabit the soil are also sensitive to contaminants (e.g., herbicides, insecticides, fungicides and heavy metals) that cause decreased or delayed growth, abnormalities of cellular metabolic pathways, and damage of cellular structures (Różalska et al. 2014; Gola et al. 2018; Szewczyk et al. 2018; Stolarek et al. 2019; Subbanna et al. 2019). Adverse changes in the cells caused by these compounds prevent these microorganisms from playing their proper ecological role. Some fungi have also developed mechanisms that allow them to survive in the presence of such contaminants (Szewczyk et al. 2018; Nowak et al. 2019).

Because of high enzymatic activity, the ability to produce secondary metabolites, and good growth in culture media, EPF are also being tested for use in other fields of biotechnology, e.g., nanoparticle biosynthesis and bioconversion of steroids or flavonoids, which are important from an economic point of view (Różalska et al. 2016; Gonzalez et al. 2017; Yosri et al. 2018; Dou et al. 2019; Kozłowska et al. 2019).

\section{Entomopathogenic fungi-short description}

Entomopathogenic fungi are parasitic microorganisms with an ability to infect and kill arthropods. They are mainly used as biopesticides in ecologic farming as a safe alternative to toxic chemical insecticides, although some of them are also used in biotechnological processes or in Chinese medicine (Jaihan et al. 2016; Ríos-Moreno et al. 2016; Lovett and Leger 2017).

Entomopathogenic fungi do not form one monophyletic group. Thus far, 12 species of Oomycetes, 65 species of Chytridiomycota, 339 species of Microsporidia, 474 species of Entomophtoromycota, 238 species of Basidiomycota, and 476 species of Ascomycota have been reported (Araújo and Hughes 2016). Species belonging to Ascomycota and Entomophtoromycota occur most frequently in nature. In the literature, species belonging to Ascomycota to the genera Metarhizium (M. anisopliae, M. robertsii, $M$. brunneum, M. lepidiotae, M. globosum, M. acridum, M. majus, M. flavoviride, M. rileyi, M. pingshaense, M. lepidiotae and M. guizhouense), Beauveria (B. bassiana and B. brongniartii), Isaria (I. fumosorosea-formerly Paecilomyces fumosoroseus, I. farinosa and I. tennuipes), Ophiocordyceps (O. sinensisformerly Cordyceps sinensis, O. unilateralis), Cordyceps (C. militaris), Torubiella (T. ratticaudata), Pochonia ( $P$. chlamydosporia), Lecanicillium ( $L$. lecani-formerly Verticillium lecanii, L. longisporum), Hirsutella (H. thompsonii, H. nodulosa, H. aphidis), and the species Paecilomyces variotii, Purpureocillium lilacinum are described (Khan et al. 2012; Tkaczuk et al. 2015; Jaihan et al. 2016.

Among the known EPF, Entomophthorales (e.g., Furia, Conidiobolus, Entomophaga, or Erynia) show the highest insecticidal activity; however, because of technical difficulties in breeding in laboratory conditions, they are not used as components of biopreparations. The most commonly used EPF are easily cultured saprotrophic fungi belonging to Ascomycota (Mascarin and Jaronski 2016). Worldwide, several different biopesticides based on species belonging to the genera Metarhizium, Beauveria, Paecilomyces, Isaria and Lecanicillium have been used. These fungi have a wide spectrum of activity, and they can therefore infect a wide variety of arthropod species (Khan et al. 2012; Castro et al. 2016; Ríos-Moreno et al. 2016).

Entomopathogenic fungi are heterogeneous organisms that play various ecological roles. For example, species of the genus Metarhizium and Beauveria, which are commonly found in soil, not only control natural arthropod populations but also form complex relationships with plants. They are described as endophytes of plant roots, stems, and leaves (Jaber and Enkerli 2017). It has been shown that $M$. robertsii and B. bassiana (but not L. lecani) provide plants with nitrogen that is assimilated during the parasitization of insects (Behie and Bidochka 2014), thus supporting plant growth (Ríos-Moreno et al. 2016). Beauveria bassiana acts as an endophyte in about 25 plant species, contributing to the control of pests and fungal plant pathogens (McKinnon et al. 2017; Vega 2018). As a fungal endophyte and epiphyte, it colonizes leaves and shoots in addition to plant roots making plants more resistant to insects (Klieber and Reineke 2016; Ramakuwela et al. 2020), and also successfully protects plants from microbial pathogens by 
suppressing disease-causing agents or increasing plant defense responses (Moonjely et al. 2016). Similarly, Lecanicillium also prevents fungal disease by growing on the surface of leaves, limiting available nutrients and producing antimicrobial compounds in addition to inducing plant responses while colonizing plant roots (Moonjely et al. 2016). Among EPF, I. fumosorosea seems not to fall into strong interactions with plants. This is probably due to the fact that Isaria spp. are sensitive to chemical substances secreted by plants and belonging to the defense system against plant pathogens (Lacey and Mercadier 1998; Zimmermann 2008). Interestingly, isolates of I. fumosorosea are reported to act against the root nematode, Meloidogyne javanica, although infection rates were found to be very low (below 40\%) (Tigano-Milani et al. 1995). EPF are primarily used in biocontrol of insect pests in the laboratory, greenhouse or in the field.

\subsection{The infectious process}

Entomopathogenic fungi infect insects by direct penetration of the cuticle. Unlike bacteria or viruses, they do not have to be ingested by an insect (Bilgo et al. 2018). The infection process starts with the adhesion of spores to arthropod shells and has two stages: the first depends on the action of hydrophobic and electrostatic forces and the second requires the activity of enzymes and low-molecular-weight proteins called hydrophobins (Skinner et al. 2014). Spore germination occurs in the presence of carbon and energy sources on the insect's cuticle at sufficient humidity and temperature. The optimum temperature for the growth and germination of EPF is between 20 and $30^{\circ} \mathrm{C}$. Spores can also germinate at temperatures outside this range and this is a characteristic feature of the particular fungal strain (Skinner et al. 2014). Subsequently, appressoria emerge, causing strong mechanical pressure on the cuticle and the production of lytic enzymes (proteo-, lipo- and chitinolytic) that disintegrate the insect's body shells (Skinner et al. 2014; Lacey et al. 2015). After penetrating the arthropod's body cavity (hemocel), the fungal hyphae start to grow. Some EPF can produce blastospores that enter host's hemolymph and produce secondary hyphae that inhabit the host's tissues. At this stage, the fungi produce secondary metabolites that cause paralysis and disrupt the host's physiological processes, mainly its immune responses (Donzelli and
Krasnoff 2016). Because of the developing infection, the insect's body is destroyed by both mechanical damage to the internal organs by the developing hyphae and nutrient depletion (Donzelli and Krasnoff 2016; Mascarin and Jaronski 2016; Fan et al. 2017).

As a result of the progressive infection, the insect's body, initially soft, becomes stiff due to fluid absorption by the fungus. Cadavers of insects attacked by fungi of the genus Beauveria may initially take on a dark red color. The entire infection process is relatively long and takes approximately 14 days after infection, but first symptoms of infection usually occur about 7 days post infection (or even earlier, depending on fungal species). After killing the insect and using all nutrition, the hyphae of the fungus emerge from the cadaver of the host through holes in its body (mouth hole, anus) and through intersegmental areas. Then, resting or infective spores are produced, which allows the fungus to spread and infect other individuals (Skinner et al. 2014).

\subsection{Infectious agents}

The pathogenesis caused by EPF requires the involvement of several infectious agents, the most important of which are adhesins, lytic enzymes, and secondary metabolites.

\subsubsection{Adhesins}

The first stage of the fungal infection process is spore adhesion to the surface of the arthropod's body. In this stage, two types of proteins are produced: hydrophobins (whose layers disintegrate during the sporulation of spores) and adhesins (MAD1 and MAD2), which enable both close adhesion to the insect's cuticle and recognition of the host by the fungal pathogen (Wang and Leger 2007; Greenfield et al. 2014).

\subsubsection{Lytic enzymes}

Lytic enzymes play the most important role during the process of insect infection by EPF. Their action, structure, and types are relatively well described. Their main role is hydrolysis of the components of the insect cuticle, which allows appressoria to penetrate the outer covers of arthropods. Lytic enzymes are 
produced as soon as the spore attaches to the cuticle and begins to form appressorium (Santi et al. 2010).

Lipases are produced first and hydrolyze the lipids and lipoproteins located in the insect epicuticle (outer cuticle) (Pedrini et al. 2007). These enzymes hydrolyze the ester bonds of triacylglycerols, which leads to the release of free fatty acids, diacylglycerols, monoacylglycerols, and glycerol (Silva et al. 2009). Moreover, lipases support the adhesion of germinating spores to insect cuticles by increasing hydrophobic interactions between the fungus and the cuticle surface (Santi et al. 2010). All EPF have been shown to contain these enzymes, most of which have been well described (Supakdamrongkul et al. 2010; Mondal et al. 2016). One of these enzymes is M. brunneum (formerly M. anisopliae var. anisopliae) phospholipase $\mathrm{C}$, which hydrolyzes phosphodiester bonds and is responsible for degrading phospholipids of insect cell membranes, which allows the fungus to enter the insect's hemocel and infect its tissues (Santi et al. 2010).

A key factor in the virulence of EPF is proteolytic enzymes that hydrolyze peptide bonds of insect cuticles, leading to the expsore of chitin fibrils. One of the first comprehensively described proteases is subtilisin (Pr1, serine endoprotease), which degrades both cuticle proteins and modifies the surface of the cuticle, thereby facilitating spore adhesion. This endoprotease is present in M. anisopliae (Shah et al. 2005), O. sinensis (Zhang et al. 2008), and B. bassiana (Donatti et al. 2008). However, other proteases such as trypsin-like acid $\operatorname{Pr} 2$ protease, cysteine Pr4 proteinase, and metalloprotease in M. anisopliae; Pr1- and Pr2like serine proteases in B. brongniartii, L. lecanii, $M$. rileyi, and Aschersonia aleyrodis; and serine elastase in Conidiobolus coronatus and B. bassiana also participate in the pathogenesis process (Zhao et al. 2016). Some studies indicate that proteases of EPF play a dual role in the infectious process. Although their main function is hydrolysis of cuticular proteins, proteases can also participate in the inactivation of antifungal proteins produced in the epidermis of insects (Sotelo-Mundo et al. 2007).

The last group of enzymes are chitinases that are classified as follows depending on the place of their action on the chitin molecule: endochitinases (hydrolyze the $\beta$-1,4-glycosidic bond inside the chitin molecule) and exochitinases (hydrolyze $N$-acetylglucosamine oligomers formed during the action of endochitinases). The combined action of endo- and exochitinases is required for the complete degradation of insect chitin. Chitinolytic enzymes have been found in numerous EPF (Duo-Chuan 2006).

Apart from proteases, lipases, and chitinases, EPF also produce other enzymes that are not directly involved in the digestion of the cuticle but play an important role in pathogenesis. One of them is acid trehalase (ATM1) that hydrolyzes trehalose, the main disaccharide of insect hemolymph, with the release of two glucose molecules, thus providing a nutrient for EPF. Inactivation of the ATM1 gene in M. acridum causes a significant reduction in the virulence of this strain, which is manifested as the inability of this species to grow inside the insect's body (Jin et al. 2015).

\subsubsection{Secondary metabolites: role in infection and possible applications}

Entomopathogenic fungi secrete abundant low-molecular-weight organic compounds called secondary metabolites, especially in response to environmental conditions. The number of produced compounds suggests that they are necessary both for maintaining the vital functions of the hosts and for effectively infecting pathogens by damaging the nervous system or reducing insect resistance (Donzelli and Krasnoff 2016). Secondary metabolites are determined using chromatographic methods combined with mass spectrometry and molecular biology techniques. On the basis of their chemical structure, they can be divided into the following groups: cyclic depsipeptides (cyclic tetradepsipeptides and cyclic hexadepsipeptides), peptides (octadepsipeptides, dipeptides, and depsipeptides), amino acid derivatives, polyketides, peptide hybrids, and terpenoids (Donzelli and Krasnoff 2016; Wang et al. 2018b) (Online resource 1). A large number of these compounds can be distinguished due to their wide occurrence and unique properties. Taking advantage of this fact, researchers keep making efforts to extend the knowledge of the mechanisms of the secondary metabolites biosynthesis and their impact on living organisms and the environment.

Destruxins (dtxs) are cyclic hexadepsipeptides isolated mainly from Metarhizium sp. More than 40 types of dtxs have been reported, of which dtxs A, B, and $\mathrm{E}$ are the most significant in the process of pathogenesis (Arroyo-Manzanares et al. 2017). Dtxs 
are well known for their insecticidal activity and phytotoxicity. They show various biological properties such as antimicrobial, antiviral, antiproliferative, cytotoxic, and immunosuppressive properties. Dtxs inhibit the action of V-ATPase proton pumps, which allows them to be used in cancer therapies or osteoporosis treatment (Liu and Tzeng 2012). However, sublines of HCT116 colon carcinoma cells are resistant to dtxs $\mathrm{A}, \mathrm{B}$, and $\mathrm{E}$ because of changes in membrane properties and reduction in ionophoric activity during increased activation of cholesterol synthesis (Heilos et al. 2018). Dtxs are also helpful in the treatment of Alzheimer's disease as they can inhibit and prevent the generation of $\beta$-amyloid (A $\beta)$ (Wang et al. 2012). On the other hand, their presence in food products is a concern as they can pose a threat to human health and disturb the balance of the ecosystem (Taibon et al. 2015). Dtxs have been found in strawberries, maize (Taibon et al. 2015), and potatoes (Carpio et al. 2016).

Beauvericins produced e.g., by $B$. bassiana, $I$. tenuipes or I. fumosorosea (Wang and Xu 2012; Weng et al. 2019) and bassianolide isolated from B. bassiana or Lecanicillum sp. are other cyclic hexadepsipeptides with interesting properties. On the one hand, beauvericins contribute to the contamination of cereal crops worldwide, bioaccumulate in different tissues, and enter the food chain by polluting eggs, milk, meat, and plant products, consequently leading to apoptosis, production of reactive oxygen species (ROS), or mitochondrial damage (Olleik et al. 2019). On the other hand, they have been shown to exert strong cytotoxic effect on tumor cells, inhibit the activity of Mycobacterium tuberculosis or Plasmodium falciparum, and restrict the activity of the cholesterol acyltransferase inhibitor (Urbaniak et al. 2019; Jajić et al. 2019). Bassianolide has activity against Staphylococcus aureus, Bacillus subtilis, Escherichia coli, Pseudomonas aeruginosa and, like beauvericins, against $M$. tuberculosis.

It inhibits several cancer cell lines and the development of the human malaria parasite Plasmodium falciparum (Keswani et al. 2019). Additionally, it inhibits acetylcholine-induced smooth muscle contraction (Xu et al. 2009).

Cordycepine, which belongs to octadepsipeptides, is a product of secondary metabolism of, among others, C. militaris and Isaria cicadae (formerly Cordyceps cicadae). It has been used in Chinese medicine for many years because of its antioxidant, anti-inflammatory, antitumor, and neuroprotective properties (Wang et al. 2019b). As cordycepine is similar to adenosine in structure, it can inhibit processes in which adenosine is involved (Xia et al. 2017). Cordycepin reduces osteoarthritis symptoms, i.e., synovial inflammation, joint pathology, and pain behavior (Ashraf et al. 2019). It has been proved that cordycepin inhibits senescence of cells in rodents through the NRF2 (nuclear factor erythroid 2-related factor 2) and AMPK (AMP-activated protein kinase) pathways in the inflammatory process. This application gives hope for the future for obviating cells aging and radiation ulcer (Wang et al. 2019b).

Oosporein isolated mainly from B. bassiana or $B$. brongniartii, a highly reactive oktadepsipeptide with insecticidal and antiviral activity, is an important secondary metabolite that can also be used as an antibiotic against gram-positive bacteria (Feng et al. 2015). The insecticidal activity of oosporein is based on the stimulation of infection rather than the direct elimination of insects. Oosporein produced by $B$. caledonica has immunosuppressive properties and contributes to greater and faster mortality of insects (Mc Namara et al. 2019). Oosporein from Cochliobolus kusanoi exerts cytotoxic effects on Madin-Darby canine kidney (MDCK) and mouse macrophage (RAW 264.7), which is mediated by ROS through oxidative stress that increases lipid peroxidation and glutathione (GSH) level (Ramesha et al. 2015).

A research study on oosporein as a siderophore in the root endophyte Chaetomium cupreum revealed that the compound enhances aluminum (Al) tolerance in the plant Miscanthus sinensis Andersson. Furthermore, it has been proved that oosporein detoxifies $\mathrm{Al}$ similar to that by the phytosiderophore chlorogenic acid (Haruma et al. 2019).

M. robertsii produces polyketides that are yellow compounds named as NG-391 and NG-393 (Donzelli and Krasnoff 2016). They have structure and properties similar to those of fusarins. Like fusarin C, NG391 exerts a strong mutagenic effect on the $\mathrm{S} 9$ fraction from rat liver in the Ames test (Donzelli et al. 2010). Further studies on these polyketides are required as they show antiproliferative effects on K-562 human leukemia cells, resulting in the impairment of the integrity of nucleic acid biosynthesis; on the other hand, their bioactivity may be related to binding to a protein involved in nucleoside phosphate biosynthesis 
or polymerization or cell death independent of caspase (Bohnert et al. 2013).

Because the secondary metabolites of EPF are a pool of compounds with diverse activity, researchers are interested in further exploring their structures, searching for new compounds, understanding their biosynthesis pathways, and conducting in-depth studies on their properties.

\section{Effect of toxic compounds on entomopathogenic fungi}

The widespread use of synthetic, anthropogenic substances has increased environmental pollution through accumulation of their residues. These toxic substances cause an adverse effect on almost all living organisms to a greater or lesser extent. Pesticides are one of the basic groups of harmful substances whose presence has been detected in soil and water. These compounds enter the soil through human activities intended to improve the growth of crops. These diverse chemical compounds are usually categorized according to their mechanism of action. Among these chemicals, herbicides (substances used to control unwanted plants), fungicides and insecticides (substances used for pest control) are the most predominant ones, and both of them have harmful effects on EPF.

There are several reports on the effects of toxic compounds related to insecticides, which are often used interchangeably with EPF (such as B. bassiana or M. anisopliae) to control pests. Organophosphate (profenophos and methyl demeton) and chloroorganic insecticides (indoxacarb) showed the highest detrimental effect on B. bassiana. Profenophos and methyl demeton inhibited B. bassiana growth by $93.72 \%$ and $95.17 \%$, respectively, after 14 days of inoculation and by $83.55 \%$ and $93.86 \%$, respectively, after 30 days of inoculation. Indoxacarb inhibited the growth of $B$. bassiana by $100 \%$ after 14 and 30 days of inoculation (Amutha et al. 2010). The organophosphate Lorsban showed the highest toxicity to M. anisopliae and $I$. fumosorosea. This compound caused more than $70 \%$ mycelial growth reduction and more than $80 \%$ conidial germination reduction in both the tested fungi (Asi et al. 2010). Insecticides belonging to carbamates can also adversely affect the growth of EPF. Carbamates (Lannate, Larvin) and a pyrrole insecticide (chlorfenapyr; Pirate) caused 40-60\% mycelia growth reduction and $50-70 \%$ conidial germination reduction in M. anisopliae and I. fumosorosea (Asi et al. 2010). The effect of the insecticide $\lambda$-cyhalothrin, belonging to pyrethroids, is less severe, and this compound at the recommended field dose caused $13.6 \%$ and $20.6 \%$ growth inhibition in $H$. nodulosa and B. bassiana, respectively (Tkaczuk et al. 2015). Among the pyrethroid insecticides, the effect of deltamethrin on B. bassiana was studied in detail (Forlani et al. 2014). Deltamethrin at $40 \mu \mathrm{g} \mathrm{cm}^{-2}$ concentration caused a significant decrease in the colony-forming unit of $B$. bassiana, but the mean inhibitory concentration $\left(\mathrm{IC}_{50}\right)$ was $50 \mu \mathrm{g} \mathrm{cm}^{-2}$. These studies also investigated the possible mechanisms of the xenobiotic's detoxification. It was observed that exposure to the xenobiotic induced two genes of cytochrome P450 (belonging to phase I detoxification) and genes of the antioxidant system such as superoxide dismutase, catalase, and glutathione S-transferase. These findings suggest that B. bassiana activates defense mechanisms in response to the harmful effects of deltamethrin.

Neonicotinoids, another group of insecticides, also have toxic effects on B. bassiana. Acetamiprid and thiamethoxam applied at average doses recommended for field application caused a significant reduction in the vegetative growth of $B$. bassiana, while the growth of $M$. anisopliae was reduced only in the presence of acetamiprid. Interestingly, among all the tested neonicotinoids, imidacloprid (highly toxic for bees and banned in many countries) did not affect germination, vegetative growth, and conidia production of the tested EPF (Neves et al. 2001). Natural insecticides are less toxic to EPF, and one of such insecticide, Spinosad (isolated from Saccharopolyspora spinosa), caused only approximately 7-8\% mycelial growth reduction and approximately $20 \%$ conidial germination reduction in M. anisopliae and I. fumosorosea (Asi et al. 2010).

Another natural insecticide, neem oil (derived from plants) was found to have a negative effect on EPF. It was revealed that it reduced the germination ratio, vegetative growth, and conidia production in $M$. anisopliae and B. bassiana (Hirose et al. 2001).

Research studies on the effects of insecticides on EPF are ongoing, and their main aim is to use lower doses of insecticides together with EPF for pest control. This task is quite important because determining a dose of an insecticide lower than that commonly applied in the field and supplementing its 
action with EPF will reduce the use of toxic compounds and significantly reduce global environmental pollution. Many studies have indicated the possibility of this synergistic action, but recently, some studies have criticized this approach (Subbanna et al. 2019).

It seems that interactions between insect pathogenic fungi and insecticides are more complicated and can have both positive and negative effects on pest control. The interaction of two botanical insecticides tondexir and palizin with B. bassiana and L. lecanii was shown. These compounds at field applied doses did not cause significant inhibition of fungal germination, but significantly reduced the susceptibility of Galleria mellonella larvae to $B$. bassiana infection. Moreover, palizin reduced the effectiveness of $L$. lecanii against G. mellonella larvae (Sohrabi et al. 2019).

The appropriate use of insecticides and fungi seems to be another important aspect. Studies conducted by Meyling et al. (2018) revealed that the synergistic effect between $\alpha$-cypermethrin and $B$. bassiana was observed only when the EPF was applied first (with an interval of $>48 \mathrm{~h}$ between applications). No synergy was observed when the insecticide was applied prior to fungal exposure within $24 \mathrm{~h}$ (Meyling et al. 2018).

A different approach was shown in other studies where only the effect of herbicides on EPF was tested and no attention was given to the effect of these compounds on the infectious potential of the fungi. The conducted research indicated that the pesticides quizalofop-p-ethyl and glyphosate, applied at the recommended field dose, caused growth inhibition of $H$. nodulosa by $63.4 \%$ and $52.1 \%$, respectively, and of B. bassiana by $43.9 \%$ and $6.5 \%$, respectively, during 20 days of cultivation (Tkaczuk et al. 2015). Among the tested herbicides, the effect of ametryn (an s-triazine herbicide) on EPF was studied most thoroughly. This compound not only inhibited growth and slowed down the degradation of glucose by $M$. brunneum but also disrupted its carbon and nitrogen metabolism. Ametryn also induced oxidative stress in the mycelia of M. brunneum (Szewczyk et al. 2018).

Another problem is the widespread use of fungicides that adversely affect all soil-borne fungi due to their mode of action. For several years, intensive research has been conducted to determine side effects of fungicides on beneficial organisms used in the Integrated Pest Management programs. Fungicides (similarly to insecticides) vary in compatibility depending on the concentration of the fungicide and the entomopathogenic fungi applied.

I. fumosorosea is sensitive to copper-based fungicides containing a higher (more than $40 \%$ ) percentage of equivalent metallic copper (Avery et al. 2013). The fungicides containing lower copper doses had no apparent impact on the growth of I. fumosorosea and $I$. farinosa but affected the infection capacity of the fungal EPF (D'Alessandro et al. 2011; Demirci et al. 2011; Avery et al. 2013). Toxic effect of copper oxide (containing 53\% and $46 \%$ metallic copper) was also confirmed for B. bassiana and L. muscarium (Kouassi et al. 2003; Ali et al. 2013).

Dithiocarbamate fungicides, harbouring metals ( $\mathrm{Zn}, \mathrm{Mn}$ ) in their molecules also have strong inhibitory effect on EPF. Mancozeb at low concentrations $\left(5 \mu \mathrm{g} \mathrm{mL}^{-1}\right.$ ) was found to inhibit I. farinosa conidia germination. This fungicide applied at average doses recommended for field application caused growth inhibition of $B$. bassiana and I. farinosa (Todorova et al. 1998; Kouassi et al. 2003). Mancozeb and propineb in low doses inhibited the sporulation of $B$. bassiana (Celar and Kos 2016). Unfortunately, mancozeb also decreased mortality of insects inoculated with EPF (Kouassi et al. 2003; Celar and Kos 2016).

Fungicide chlorothalonil (a broad-spectrum of fungicide, widely used, nitro compound) seems to have little effect on B. bassiana growth. However, it was shown that this fungicide severely inhibited conidial germination of $I$. farinosa and B. bassiana even at a low concentration of $5 \mu \mathrm{g} \mathrm{mL}^{-1}$ (Demirci et al. 2011; Celar and Kos 2016). In other studies chlorothalonil in field doses demonstrated fungicidal activity against $B$. bassiana and $M$. anisopliae by the inhibition or reduction of growth and sporulation (Fiedler and Sosnowska 2017). This fungicide also inhibited germination of $L$. muscarium spores (at concentration $10 \mathrm{ug} \mathrm{mL}^{-1}$ ) but unexpectedly it did not suppress chitinase, $\operatorname{Pr} 1, \operatorname{Pr} 2$ and lipase activity suggesting its possible compatibility with the fungus (Bagga et al. 2004). Similarly to chlorothalonil, another widely used fungicide captan (dicarboximide) only slightly hindered mycelial growth but severely inhibited conidial germination B. bassiana, I. fumosorosea, L. longisporum (Shah et al. 2009; Dara 2017). Captan applied in field doses with $B$. bassiana severely affected the mortality in T. molitor larvae (Dara 2017).

Triazole fungicides have a more diverse effect on EPF and among them the most toxic is tebuconazole. 
This compound suppressed the growth of B. bassiana and $M$. anisopliae at concentration $1000 \mathrm{ppm}$ and $L$. lecanii at concentration $100 \mathrm{ppm}$. Other triazole fungicides, difenaconazole, tricyclazole and myclobutanil were also toxic to L. lecanii and L. muscarium (Demirci et al. 2011; Reddy et al. 2018). Tebuconazole was highly toxic to I. farinosa (at concentration $1 \mu \mathrm{g} \mathrm{mL}^{-1}$ ) and to $P$. lilacinus (at $3 \mu \mathrm{g} \mathrm{mL}^{-1}$ ) causing both conidial germination and mycelial growth inhibition (Demirci et al. 2011). This fungicide also hindered the entomopathogenic activity of $I$. farinosa in all three life stages of the mealybug (Demirci et al. 2011) and completely inhibited the entomopathogenic activity of the fungus Erynia neoaphidis on pea aphid (Acyrthosiphon pisum) (Latteur and Jansen 2002).

Azoxystrobin (strobilurin fungicide) was found to have a negative impact on growth and sporulation of B. bassiana and $M$. anisopliae and to be highly deleterious to conidial production and growth of $I$. fumosorosea (D'Alessandro et al. 2011; Fiedler and Sosnowska 2017). It exhibited moderate toxicity toward M. majus (growth inhibition by $40 \%$, sporulation reduction by $60 \%$ ) compared to other common fungicides used in banana agro-system (Sivakumar et al. 2019). However, the influence of azoxystrobin on EPF virulence varied. This compound did not affect the insect infection by B. bassiana, whereas it caused a significant inhibition of insect infection by I. fumosorosea (Shah et al. 2009).

Recent scientific research suggests that EPF sensitivity to fungicides can be reduced by using microsclerotia instead of spores alone. Chlorothalonil and iprodione, seem to have a limited impact on the ability of microsclerotia of $M$. brunneum to grow out and produce viable conidia, compared to their impact on germination of conidia and growth of mycelium (Wu et al. 2020). Some fungicides may need to be applied some time before or after the application of EPF to minimize any suppressive effects. However, the benefit of separating the applications in time needs to be investigated in future studies.

The effects of other anthropogenic compounds such as endocrine disrupting compounds (EDCs) on EPF are relatively rarely studied. It was found that nonylphenols are toxic to Metarhizium. These compounds at high concentrations (50 and $100 \mathrm{mg} \mathrm{L}^{-1}$ ) caused severe changes in the pellet morphology of $M$. robertsii and reduced its metabolic activity. These disruptions were associated with changes in the hyphae structure that were manifested in terms of increased vacuolization, an increase in the size and change in the shape of mitochondria, and destruction of the plasma membrane (that leads to autolysis). Changes were also noted in the thickness and composition of the cell wall (Różalska et al. 2014). Another EDC - dibutyltin - and the by-product of its dealkylation, monobutyltin, caused nitrosative and oxidative stress and changes in the lipid profiles of $M$. robertsii (Siewiera et al. 2017a; Stolarek et al. 2019).

Industrial dyes can also adversely affect the growth of EPF. Reactive remazol red, Indanthrene blue, Yellow 3RS, and Vat Novatic Grey caused a reduction in mycelium biomass. Yellow 3RS showed the lowest adverse effect on the growth of $B$. bassiana. In the presence of this dye, the amount of biomass obtained was $5.3 \mathrm{~g} \mathrm{~L}^{-1}$. Reactive remazol red and Indanthrene blue were more harmful to $B$. bassiana than Yellow 3RS. Among the tested dyes, Vat Novatic Grey was the most toxic to B. bassiana MTCC 4580 strain (Gola et al. 2018).

Other anthropogenic substances such as biofertilizers, which seem to be harmless to the environment, may also affect the growth of soil-inhabiting EPF. Biofertilizers are products applied on the surface of a plant or in soil and contain live microorganisms that promote plant growth and development. These products may include bacterial species such as Rhizobium, Azotobacter, and Azospirilium as well as blue green algae (BGA) (Kumar et al. 2017). Hirose et al. (2001) determined the effect of three biofertilizers (E.M.-4, Multibion $^{\mathrm{TM}}$, and Supermagro) on two species of EPF: M. anisopliae and B. bassiana. E.M.-4 and Multibion $^{\mathrm{TM}}$ reduced the germination ratio, vegetative growth, and conidia production in $M$. anisopliae. Multibion $^{\mathrm{TM}}$ was found to be moderately toxic to $B$. bassiana (Hirose et al. 2001).

\section{Removal of toxic pollutants by entomopathogenic fungi}

Entomopathogenic fungi are commonly found in natural environment, where they come in contact with various anthropogenic pollutants. Although the mechanisms regulating the pathogenesis of insects by EPF have been relatively well understood, only a few studies have reported the interaction of toxic 
impurities with these microorganisms. One of the most harmful groups of anthropogenic compounds are EDCs. They are natural or synthetic substances which, because of their structural similarity and ability to bind to hormone receptors in the cell, can disrupt the functioning of the human and animal endocrine system. This group includes, among others, alkylphenols (e.g., nonylphenol), organotin compounds (tributyl tin [TBT]), synthetic estrogens (e.g., 17 $\alpha$ ethinylestradiol [EE2]), and pesticides (e.g., ametryn) (Różalska et al. 2015; Szewczyk et al. 2018; Nowak et al. 2019; Stolarek et al. 2019).

\subsection{Removal of 4-n-nonylphenol by entomopathogenic fungi}

Nonylphenols are xenoestrogens widely used in the manufacture of many household products. The most commonly used nonylphenols are para-nonylphenols (4-nonylphenols [4-NP]), which occur in the form of several dozen isomers differing in the degree of branching of the alkyl fragment of the molecule. These compounds are extremely harmful for the natural environment because of their high toxicity to aquatic organisms and the ability to accumulate. They enter the environment primarily as biodegradable products of surfactants and detergents. 4-NPs also disrupt the functioning of the human and animal endocrine system by imitating $17 \beta$-estradiol and binding to estrogen receptors. These compounds have a negative effect on microorganisms and soil invertebrates and restrict plant growth (Acir and Guenther 2018).

Strains of the genus Metarhizium can degrade the unbranched isomer of nonylphenol (4-n-NP). $M$. robertsii and $M$. brunneum showed the highest activity in cultures after $24 \mathrm{~h}$ incubation with the compound, with more than $90 \%$ loss of substrate (Różalska et al. 2013). The extracts of $M$. robertsii culture showed 35 derivatives that were formed after the degradation of this xenobiotic (Różalska et al. 2015; Nowak et al. 2019). The proposed degradation pathway indicates that the fungi of the genus Metarhizium have unique capabilities to perform numerous, simultaneous mono- and dihydroxylation reactions both in the aliphatic chain and the 4- $n$-NP aromatic ring (Nowak et al. 2019). In addition, these fungi completely degraded nonylphenol in the culture environment to form $\mathrm{CO}_{2}$ and $\mathrm{H}_{2} \mathrm{O}$ (Różalska et al. 2015; Nowak et al. 2019).
4.2 Triazine removal by entomopathogenic fungi

Triazines are herbicides that possess a heterocyclic ring containing three unsaturated nitrogen atoms. These compounds have a carcinogenic effect and disrupt the functioning of the endocrine system in living organisms; therefore, their use is prohibited in the European Union, although their presence is still found in the natural environment. The conducted studies showed the ability of M. brunneum to partially decompose ametryn, a triazines, at an initial concentration of $100 \mathrm{mg} \mathrm{L}^{-1}$ to generate four derivatives (Szewczyk et al. 2018).

\subsection{Dibutyltin removal by entomopathogenic fungi}

Dibutyltin (DBT) is used as a curing agent for silicone rubbers and as a catalyst in esterification reactions. It enters the environment through polyvinyl chloride (PVC) materials and as a degradation product of TBT. DBT exhibits immunotoxic, neurotoxic, and prooxidative properties. Aerated cultures of $M$. robertsii can completely biodegrade DBT (added at an initial concentration of $20 \mathrm{mg} \mathrm{L}^{-1}$ ) to monobutyltin (MBT) and hydroxylated MBT. The addition of vitamin $\mathrm{C}$ caused a decrease in oxidative stress associated with the presence of tin compounds in the medium, and the tested strain in the presence of this vitamin degraded MBT threefold faster (Siewiera et al. 2017a). The presence of natural estrogens - estrone (E1) and 17 $\beta$ estradiol (E2) — accelerated the degradation of DBT. Moreover, both the tested estrogens protected EPF against oxidative stress caused by the presence of MBT in the culture medium (Siewiera et al. 2017b).

The cell membrane fluidity of $M$. robertsii raises the possibility of this EPF to degrade toxic substances. A membrane with a less compact structure, and thus with higher permeability, allows the transport of toxic compounds into the cell, where they are degraded (Stolarek et al. 2019).

\subsection{Synthetic estrogen removal by entomopathogenic fungi}

EE2 is a synthetic estrogen ubiquitously present in the environment (soil, groundwater, rivers, lakes, and seas) because of its widespread use and resistance to biodegradation. It is a derivative of $\mathrm{E} 2$ and is used in 
hormonal contraception. Various strains of $M$. robertsii can partially remove EE2 from the growth medium with significant efficiency, which reaches up to $85 \%$ (Różalska et al. 2015).

\subsection{Removal of hydrocarbons ( $n$-alkanes) by entomopathogenic fungi}

Entomopathogenic fungi can assimilate $n$-alkanes. The metabolic pathways of the decomposition of these compounds and the enzymes involved in this process are relatively well known (Keyhani 2018). These hydrocarbons are xenobiotics commonly found in the environment; they are also found in small amounts in the insect epicuticle, which, together with the wax layer, is the first important barrier to overcome for EPF. Lipids containing long hydrocarbon chains are present in all external insect layers. The fungus $B$. bassiana can use $n$-alkanes (extracted from insect surfaces) as a carbon source. These compounds are first hydroxylated by cytochrome $\mathrm{P} 450$ present in the microsomal fraction and then catabolized by $\beta$-oxidation. This fungus can also grow and metabolize synthetic straight-chain alkanes (up to $n$-C33) (Huarte-Bonnet et al. 2015). Other EPF, e.g., $M$. robertsii, are also capable of using these linear hydrocarbons (e.g., $n$-hexadecane or $n$-octacosan) as the only source of carbon and energy, and one of the first stages of degradation is carried out by cytochrome P450 (Huarte-Bonnet et al. 2018).

\subsection{Removal of industrial dyes by entomopathogenic fungi}

Gola et al. (2018) tested the ability of the EPF $B$. bassiana MTCC 4580 to remove four industrial dyes (Reactive remazol red, Indanthrene blue, Yellow 3RS, and Vat Novatic Grey) from wastewater. The strain $B$. bassiana MTCC 4580 could eliminate the dyes (added to the cultures at a high initial concentration of $500 \mathrm{mg} \mathrm{L}^{-1}$ ) with high efficiency, which reached even 97\%. Maximum removal was observed for Indanthrene blue (97\%), while the removal of Vat Novatic Grey and Reactive remazol red was $91 \%$ and $84 \%$, respectively. The mechanism of synthetic dye removal by $B$. bassiana is based on bioaccumulation and not on biodegradation (Gola et al. 2018).

\section{Influence of heavy metals on entomopathogenic fungi}

Entomopathogenic fungi can be used for heavy metal removal from the environment by improving the phytoremediation properties of plants (Farias et al. 2019). Even trace amounts of metals in the environment can have an adverse effect on soil, the ecosystem, water circulation, and elements in the environment as well as on human and animal health. The presence of heavy metals in the human body can contribute to the development of autoimmune diseases, digestive system disorders, and diseases of many important internal organs, including the heart, liver, kidneys, and lungs. An increase in heavy metal occurrence in the environment is due to the development of mining and electroplating industries, vehicular traffic, and waste (Hussein et al. 2011; Gola et al. 2016). The removal of heavy metal residues from the environment poses problems because of their tendency to accumulate in soil. The use of physicochemical methods of metal removal is expensive and unfavorable for the environment and uneconomic when the metal concentration is low.

The EPF $B$. bassiana has confirmed ability to remove heavy metals from contaminated water. This fungal species can remove several heavy metals such as $\mathrm{Cu}$ (II), Ni (II), Cd (II), Zn (II), Cr (VI), and Pb (II) added at the initial concentration of $30 \mathrm{mg} \mathrm{L}^{-1}$ with good efficiency ranging from $58 \%$ to $75 \%$. This study also revealed the bioaccumulation of $\mathrm{Pb}$ (II) inside $B$. bassiana cells, although the exact mechanism of this phenomenon has not yet been proposed (Gola et al. 2018). Lead was also found to cause severe morphological alterations such as an increase in surface roughness and hyphae density and aggregation. It was also found that heavy metals are not very toxic to $B$. bassiana and the minimum inhibitory concentrations (MIC) were as follows: $\mathrm{Zn}$ (II): $250 \mathrm{mg} \mathrm{L}^{-1}$; Cd (II): $200 \mathrm{mg} \mathrm{L}^{-1}$; $\mathrm{Cu}$ (II), Ni (II) and Cr (VI): $100 \mathrm{mg} \mathrm{L}^{-1}$ (Gola et al. 2018).

The mechanism of $B$. bassiana susceptibility to $\mathrm{Cr}$ (III) has been partly explained by the studies on the role of the aldo-keto reductase Bdakr1 (Wang et al. 2018b). Like other heavy metals, chromium causes oxidative stress in fungal cells and intracellular lipid peroxidation, which leads to the accumulation of malonaldehyde (MDA). Under the normal condition, intracellular MDA levels were similar between the 
wild type and $\Delta \mathrm{Bbakr} 1$. The presence of the heavy metal $\left(5 \mathrm{mM} \mathrm{Cr}\left(\mathrm{NO}_{3}\right)_{3}\right)$ in the culture medium caused a significant increase in MDA levels in both the wild type and $\Delta$ Bbakr 1 cells, but in the case of the mutant, the levels were significantly higher than those in the wild type. A growth reduction of $\Delta$ Bbakr1 strain was also noted. These data suggest an impaired ability of the mutant cells to detoxify the heavy metal by catalyzing reduction reactions of aldehydes or ketones to primary and secondary alcohols (Wang et al. 2018a).

The biomass of two EPF (B. bassiana AUMC 3263 and $M$. anisopliae AUMC 3085) can also serve as an efficient biosorbent for $\mathrm{Pb}$ (II) and $\mathrm{Cd}$ (II) from aqueous metal solutions. The maximum lead adsorption capacities of B. bassiana and M. anisopliae were 83.33 and $66 \mathrm{mg} \mathrm{g}^{-1}$, respectively, while for cadmium, these values reached 46 and $44 \mathrm{mg} \mathrm{g}^{-1}$, respectively. The higher adsorption capacity of $B$. bassiana biomass than that for M. anisopliae could be due to the differences in the cell wall structure. The highest rate of metal absorption was observed during the first $5 \mathrm{~min}$ of the experiment and reached a constant value after 30 min (Hussein et al. 2011).

Heavy metals can also negatively affect the growth of EPF. Tkaczuk et al. (2019) determined the effect of five heavy metals (Zn (II), Ni (II), Cu (II), Cd (II), and $\mathrm{Pb}$ (II)) on the growth of I. farinosa, I. fumosorosea, and I. tennuipes. The experiments were conducted on Sabouraud solid medium supplemented with heavy metals at concentrations that normally occur in soil (i.e., $\mathrm{Zn}$ (II): 33.0; $\mathrm{Pb}$ (II): 13.8; $\mathrm{Cu}$ (II), $\mathrm{Ni}$ (II): 6.5; Cd (II): $0.22 \mathrm{mg} \mathrm{L}^{-1}$ ) and at concentrations that were 10 and 100 times higher. The obtained data revealed that the greatest toxic effect of heavy metal ions on the EPF was observed when their concentration was 100 times higher than the natural content (on average, approximately $50.5 \%$ inhibition of growth). The greatest inhibitory effect on the growth of the fungal colonies was showed by $\mathrm{Ni}$ (II), while $\mathrm{Pb}$ (II) showed the least effects. Among all the tested species, I. fumosorosea had the highest tolerance to the presence of heavy metals.

Plants are widely used for bioremediation of heavy metal-contaminated areas. They interact closely with microorganisms, which can enhance plant growth and health by increasing nutrient uptake and improving plant resistance to pathogens and stress (Kidd et al. 2017). EPF are not only insect pathogens, but they are also associated with plant rhizosphere, thereby promoting plant growth. Farias et al. (2019) investigated the effect of fungal consortium containing four species of EPF (B. bassiana, M. anisopliae, P. chlamydosporia, and $P$. lilacinum) on the ability of Jacaranda mimosifolia to absorb $\mathrm{Cu}$ (II), Zn (II), and Mn (II). After 45 days of experiments, it was found that the use of the fungal preparation in combination with biochar caused an increase in the mass of roots and shoots of $J$. mimosifolia, intensified the translocation of metals from roots to shoots, and inhibited the leaching of metals from plant tissues, which resulted in an increased concentration of these metals in J. mimosifolia tissues. The application of the consortium of fungal isolates and biochar improved the phytoremediation potential of J. mimosifolia.

\section{Nanoparticle biosynthesis by entomopathogenic fungi}

During the last decade, metal nanoparticles have received considerable attention due to their wide range of applications in many different fields (e.g., pest management). They have also been used as antimicrobial agents and biomarkers. The production of nanoparticles by fungi is an interesting and ecological alternative to chemical and physical syntheses. Extracts from EPF or their culture media containing numerous enzymes can also serve as catalysts for the production of nanoparticles.

The waste mycelium after biodegradation processes is a valuable source of both enzymes and many other substances that can be reused in various processes. An example is the use of $M$. robertsii waste biomass (obtained after nonylphenol degradation) for the production of silver nanoparticles (AgNPs). Aqueous extracts from the waste mycelium $M$. robertsii, in dilutions of $25 \%$ and $50 \%$, allowed the synthesis of silver nanoparticles from silver nitrate, and the obtained nanoparticles were small in size (from 2 to $29 \mathrm{~nm}$ ) and relatively monodisperse, which is an important factor in their later use. The obtained colloids also exhibited antimicrobial activity, and the addition of $30 \mathrm{ppm}$ AgNPs caused growth inhibition of $S$. aureus and E. coli (Różalska et al. 2016). These nanoparticles were also found to have fungicidal properties against Candida albicans, C. glabrata, and C. parapsilosis minimum inhibitory concentration 
(MIC) values ranged from 1.56 to $6.25 \mu \mathrm{g} \mathrm{mL}^{-1}$. An $85 \%$ decrease in viability of the tested yeast was observed after 6 h of incubation with AgNPs (added at a concentration of $6.25 \mu \mathrm{g} \mathrm{mL}^{-1}$ ); it was also observed that this concentration of nanoparticles was not toxic to mammalian cells. The main mechanism of action of AgNPs against pathogenic yeast is probably based on changes in membrane permeability associated with a decrease in the amount of C18:2 phospholipids (Różalska et al. 2018).

In developing countries, the major public health concerns are vector-borne diseases. EPF are frequently used in protection against mosquitoes. Moreover, nanoparticles produced by these microorganisms were also found to be effective in biocontrol programs.

Myco-synthesized AgNPs are also a potential larvicidal and eco-friendly agent for controlling mosquito population. AgNPs obtained from M. anisopliae culture extracts may be relevant as biocontrol agents for the malaria vector Anopheles culicifacies mosquitos. The $\mathrm{LC}_{50}$ (median lethal concentration) of the studied nanoparticles ranged from 32 to $51 \mathrm{mg} \mathrm{L}^{-1}$ for various larval stages and $60 \mathrm{mg} \mathrm{L}^{-1}$ for pupae, although the low doses of silver nanoparticles limited the development of larvae and pupae (Amerasan et al. 2016). AgNPs synthesized by $I$. fumosorosea are even more effective against mosquitos. These nanoparticles were found to be more susceptible against all larval instars of Culex quinquefasciatus and Aedes aegypti. The mortality of all larval instars in the presence of $1 \mathrm{ppm}$ of AgNPs ranged from $92 \%$ to $100 \%$. The $\mathrm{LC}_{50}$ and $\mathrm{LC}_{90}$ values were $0.240-0.652$ and $1.219-2.916$, respectively, for all larval instars (I-IV) of $C$. quinquefasciatus and 0.065-0.137 and 0.558-1.278 ppm for A. aegypti (Banu and Balasubramanian 2014). The filtrates of $I$. fumosorosea can also produce iron nanoparticles that can serve as an insecticide against sweet potato whitefly Bemisia tabaci. These nanoparticles showed high pathogenicity against nymphs (second and third instar) and pupae, with $\mathrm{LC}_{50}$ values ranging from 19.17 to $37.71 \mathrm{ppm}$. $\mathrm{LT}_{50}$ (lethal time) at the iron nanoparticle concentration of $50 \mathrm{ppm}$ was shorter for second instar nymphs (3.15 days) and longer for pupae (4.22 days) (Wang et al. 2019a).

Metarhizium anisopliae-synthesized titanium nanoparticles (TiNPs) also have insecticidal potential. The larvicidal activity of TiNPs against G. mellonella was $22 \%$, but the synergistic effect of these nanoparticles and gamma-irradiated $M$. anisopliae (with higher lipase, protease, amylase, and nitrate reductase activities) caused $82 \%$ larval mortality (Yosri et al. 2018).

\section{Biotransformation performed using entomopathogenic fungi}

Entomopathogenic fungi can perform biotransformation, leading to the generation of derivatives with higher activity. These processes are favorable, because they improve the absorbability of products (Yang et al. 2018; Dou et al. 2019; Kozłowska et al. 2019).

\subsection{Steroid biotransformation}

Steroids are compounds with high economic importance, and they are widely used in medicine as hormones and active drugs. Compounds of pharmaceutical significance are obtained by modification and transformation. Because chemical methods of steroid modification are very complicated and involve even several stages, transformations are most often performed using microorganisms. Microbiological transformations of steroids include hydroxylation, BaeyerVilliger oxidation, reduction, and isomerization.

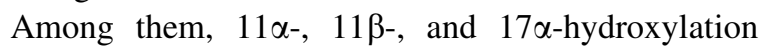
reactions carried out by filamentous fungi are the key reactions to obtain molecules with high biological activity (Xiong et al. 2006; Gonzalez et al. 2017; Kozłowska et al. 2019).

Many filamentous fungi are used in steroid transformations, and EPF can also be efficiently used for transformations. Recent studies have shown that $12 I$. farinosa strains could effectively transform dehydroepiandrosterone (DHEA) to $7 \alpha$ - and $7 \beta$-hydroxy derivatives. One of these strains, I. farinosa $\mathrm{KCh}$ $\mathrm{KW} 1.1$, transformed androstenediol, androstenedione, adrenosterone, $17 \alpha$-methyltestosterone, 17 $\beta$-hydroxyandrost-1,4,6-triene-3-one, and progesterone to mono or dihydroxy derivatives with a high yield and stereoselectivity (Kozlowska et al. 2019).

In another study, biotransformation of estrogens (E1, E2, and EE2) by I. fumosorosea was confirmed. Most of the estrogens were hydroxylated at C-6 position and glycosylated at C-3 position. The presence of a methyl group at the $\mathrm{C}-3$ steroid ring 
completely inhibited the glycosylation of estrogens by I. fumosorosea, indicating that a free hydroxyl group at this position is necessary for glycosylation (Kozłowska et al. 2019).

Entomopathogenic fungi can also transform steroids on a large scale, which can be used for industrial purpose in the future. $11 \alpha$-Hydroxy was transformed to 13-ethyl-gon-4-ene-3,17-dione (GD, a key step in the synthesis of oral contraceptive desogestrel) by $M$. anisopliae and nano-liposome technique with a high yield. The main reaction product $11 \alpha$-hydroxy-13ethyl-gon-4-ene-3,17-dione (HGD) was also produced at high concentration of GD as $6 \mathrm{~g} \mathrm{~L}^{-1}$ with no reduction of transformation efficiency. The main byproduct of the reaction $6 \beta, 11 \alpha$-dihydroxy derivative was found to benefit the purification of HGD (Feng et al. 2014).

Beauveria bassiana may also find applications in the pharmaceutical industry because it gives high yields of valuable $11 \alpha$-hydroxy intermediates of steroids (Gonzalez et al. 2017). This process, however, seems to require optimization. In $B$. bassiana culture media with androst-4-ene-3,17-dione at $\mathrm{pH}=6.0$, only two hydroxylated metabolites were found, while in samples at $\mathrm{pH}=7.0$, four hydroxylated and/or reduced metabolites were detected. These data show that B. bassiana is clearly an efficient biocatalyst for $11 \alpha$-hydroxylation and reduction of the 17-carbonyl group (Xiong et al. 2006). B. bassiana strain ATCC7159 transforms DHEA to $3 \beta, 11 \alpha, 17 \beta$-trihydroxyandrost-5-ene in the presence of a moderate DHEA concentration $\left(0.83-1.0 \mathrm{~g} \mathrm{~L}^{-1}\right)$ and in a mild environment $\left(26^{\circ} \mathrm{C}, \mathrm{pH} 7\right)$ (Gonzalez et al. 2017). The addition of various compounds can positively affect the biosynthesis of these pharmaceutical metabolites. An example is the enhanced hydroxylation capacity of $B$. bassiana with $n$-alkane solvents as a carbon source. The addition of n-dodecane (n-C12) and n-hexadecane (n-C16) was found to enhance the conversion of DHEA to $3 \beta, 11 \alpha, 17 \beta$-trihydroxyandrost-5-ene, resulting in the appearance $22.8 \% \mathrm{mM}$ hydroxylated product in the culture medium. An unusual feature of Beauveria is the mode of conducting the reaction, which consists of the reduction of the $\mathrm{C}-17$ ketone of DHEA preceded by $11 \alpha$-hydroxylation. This phenomenon differs from the reaction carried out by other microorganisms, which first activate an unfunctionalized carbon to $11 \alpha$-hydroxy-17-oxo derivative before obtaining a $3 \beta, 11 \alpha, 17 \beta$-triol product (Gonzalez et al. 2015).

\subsection{Flavonoid biotransformation}

Flavonoids are derivatives of plant polyphenols. These are glycosides with $\mathrm{O}$ - and $\mathrm{C}$-glycosidic bonds connecting the base with sugar residues (Dymarska et al. 2018a, b; Dou et al. 2019), including most often glucose, rhamnose, galactose, xylose, and arabinose (Yang et al. 2018). It is believed that the sugar group of flavonoids is the main factor affecting the absorption of these compounds by the human body (Sordon et al. 2017). There are six classes of flavonoids: flavones, isoflavones, flavanones, flavanols, flavanonols, and flavan-3-ols. Because of their antioxidant, anti-inflammatory, and antibacterial properties, they exert beneficial effects on human health. In addition, flavonoids have anti-tumor and immunomodulatory activity and protective effects on the human nervous system. The consumption of foods containing flavonoids can prevent the development of heart disease, diabetes, and depression (Dymarska et al. 2018a; Yang et al. 2018; Dou et al. 2019). They occur naturally in vegetables, fruits, cereals, wine, and tea (Yang et al. 2018; Dou et al. 2019).

Microorganisms can efficiently biotransform flavonoids to flavonoid glycosides, which are better absorbed than flavonoids themselves. Among microorganisms, EPF seem to have great potential in these processes; however, currently, Isaria and Beauveria are studied frequently, and the potential of Metarhizium remains undiscovered. The relevant data are presented in Table 1.

\section{Conclusions}

A review of literature showed that in addition to the use of EPF in biocontrol, these fungi can also be used in different ways that have not yet been described in previous reviews. EPF can also be used for removing harmful substances from the environment, because they possess enzymes that enable the removal of toxic compounds of anthropogenic origin. For example, $M$. robertsii and $M$. brunneum species can degrade nonylphenols, triazines, tin compounds, synthetic estrogen, hydrocarbons, and even industrial dyes. These microorganisms also interact with heavy 


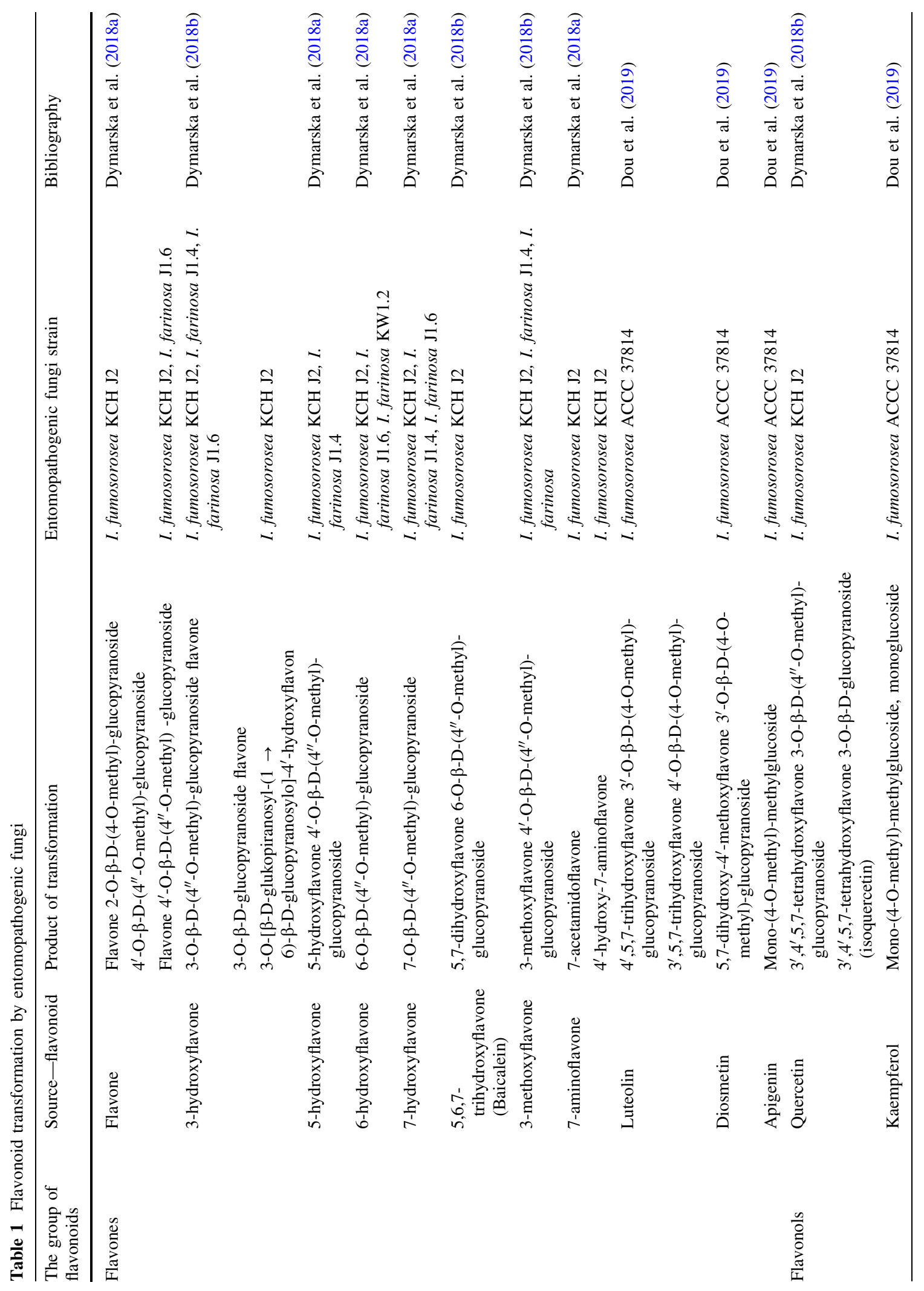




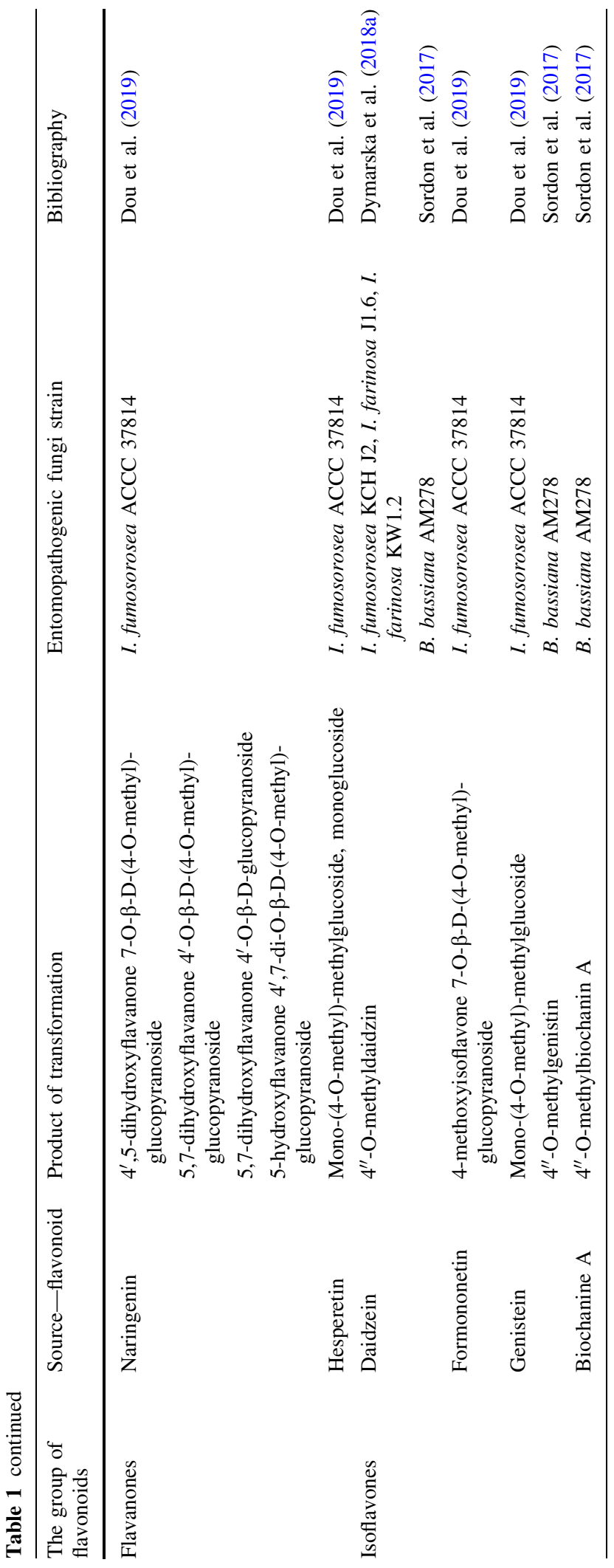


metals, which sometimes adversely affect their growth or hyphae morphology; however, these fungi possess the ability to remove heavy metals from the environment, for example, from contaminated waters (zinc, copper, cadmium, nickel, and chromium) and indirectly by improving the phytoremediation properties of plants (copper, manganese, and zinc). On the basis of this current review article, the authors conclude that EPF have great potential in the biosynthesis of biologically active compounds used in medicine, for example, hydroxylated steroids and glycated flavonoids or for bioproduction of silver nanoparticles with antibacterial and antifungal properties. Because of the high potential shown by EPF and the many different functions that they can perform, they are being increasingly researched.

Acknowledgements This research was financed by a grant from the National Science Center in Krakow (Poland), Contract Number UMO-2016/23/B/NZ9/00840.

\section{Compliance with ethical standards}

Conflict of interest The authors declare that they have no conflict of interest.

Open Access This article is licensed under a Creative Commons Attribution 4.0 International License, which permits use, sharing, adaptation, distribution and reproduction in any medium or format, as long as you give appropriate credit to the original author(s) and the source, provide a link to the Creative Commons licence, and indicate if changes were made. The images or other third party material in this article are included in the article's Creative Commons licence, unless indicated otherwise in a credit line to the material. If material is not included in the article's Creative Commons licence and your intended use is not permitted by statutory regulation or exceeds the permitted use, you will need to obtain permission directly from the copyright holder. To view a copy of this licence, visit http://creativecommons.org/licenses/by/4.0/.

\section{References}

Acir IH, Guenther K (2018) Endocrine-disrupting metabolites of alkylphenol ethoxylates-a critical review of analytical methods, environmental occurrences, toxicity, and regulation. Sci Total Environ 635:1530-1546. https://doi.org/ 10.1016/j.scitotenv.2018.04.079

Ali S, Huang Z, Ren S (2013) Effect of fungicides on growth, germination and cuticle-degrading enzyme production by Lecanicillium muscarium. Biocontrol Sci Technol 23:711-723. https://doi.org/10.1080/09583157.2013. 794258
Amerasan D, Nataraj T, Murugan K et al (2016) Myco-synthesis of silver nanoparticles using Metarhizium anisopliae against the rural malaria vector Anopheles culicifacies Giles (Diptera: Culicidae). J Pest Sci 89:249-256. https:// doi.org/10.1007/s10340-015-0675-x

Amutha M, Gulsar Banu J, Surulivelu T, Gopalakrishnan N (2010) Effect of commonly used insecticides on the growth of white Muscardine fungus, Beauveria bassiana under laboratory conditions. J Biopestic 3:143-146

Araújo JPM, Hughes DP (2016) Diversity of Entomopathogenic Fungi Which groups conquered the insect body? In: Lovett B, Leger RJS (eds) Advances in genetics, vol 94. Elsevier, Amsterdam, pp 1-39. https://doi.org/10.1016/bs.adgen. 2016.01.001

Arroyo-Manzanares N, Diana Di Mavungu J, Garrido-Jurado I et al (2017) Analytical strategy for determination of known and unknown destruxins using hybrid quadrupole-Orbitrap high-resolution mass spectrometry. Anal Bioanal Chem 409:3347-3357. https://doi.org/10.1007/s00216-0170276-Z

Ashraf S, Radhi M, Gowler P et al (2019) The polyadenylation inhibitor cordycepin reduces pain, inflammation and joint pathology in rodent models of osteoarthritis. Sci Rep 9:4696. https://doi.org/10.1038/s41598-019-41140-1

Asi MR, Bashir MH, Afzal M et al (2010) Compatibility of entomopathogenic fungi, Metarhizium anisopliae and Paecilomyces fumosoroseus with selective insecticides. Pak J Bot 42:4207-4214

Avery PB, Pick DA, Aristizábal LF et al (2013) Compatibility of Isaria fumosorosea (Hypocreales: Cordycipitaceae) blastospores with agricultural chemicals used for management of the Asian citrus psyllid, Diaphorina citri (Hemiptera: Liviidae). Insects 4:694-711. https://doi.org/10.3390/ insects4040694

Bagga S, Hu G, Screen SE, Leger RJS (2004) Reconstructing the diversification of subtilisins in the pathogenic fungus $M e$ tarhizium anisopliae. Gene 324:159-169. https://doi.org/ 10.1016/j.gene.2003.09.031

Banu AN, Balasubramanian C (2014) Optimization and synthesis of silver nanoparticles using Isaria fumosorosea against human vector mosquitoes. Parasitol Res 113:3843-3851. https://doi.org/10.1007/s00436-0144052-0

Behie SW, Bidochka MJ (2014) Ubiquity of insect-derived nitrogen transfer to plants by endophytic insect-pathogenic fungi: an additional branch of the soil nitrogen cycle. Appl Environ Microbiol 80:1553-1560. https://doi.org/10.1128/ AEM.03338-13

Bilgo E, Lovett B, Leger RJS et al (2018) Native entomopathogenic Metarhizium spp. from Burkina Faso and their virulence against the malaria vector Anopheles coluzzii and non-target insects. Parasites Vectors 11:11-16. https://doi.org/10.1186/s13071-018-2796-6

Bohnert M, Dahse HM, Gibson DM et al (2013) The fusarin analog NG-391 impairs nucleic acid formation in K-562 leukemia cells. Phytochem Lett 6:189-192. https://doi.org/ 10.1016/J.PHYTOL.2013.01.001

Carpio A, Arroyo-Manzanares N, Ríos-Moreno A et al (2016) Development of a QuEChERS-based extraction method for the determination of destruxins in potato plants by 
UHPLC-MS/MS. Talanta 146:815-822. https://doi.org/ 10.1016/j.talanta.2015.06.008

Castro T, Mayerhofer J, Enkerli J et al (2016) Persistence of Brazilian isolates of the entomopathogenic fungi $\mathrm{Me}$ tarhizium anisopliae and $M$. robertsii in strawberry crop soil after soil drench application. Agric Ecosyst Environ 233:361-369. https://doi.org/10.1016/j.agee.2016.09.031

Celar FA, Kos K (2016) Effects of selected herbicides and fungicides on growth, sporulation and conidial germination of entomopathogenic fungus Beauveria bassiana. Pest Manag Sci 72:2110-2117. https://doi.org/10.1002/ps.4240

D’Alessandro CP, Padin S, Urrutia MI, López Lastra CC (2011) Interaction of fungicides with the entomopathogenic fungus Isaria fumosorosea. Biocontrol Sci Technol 21:189-197. https://doi.org/10.1080/09583157.2010. 536200

Dara SK (2017) Compatibility of the entomopathogenic fungus Beauveria bassiana with some fungicides used in California strawberry. Open Plant Sci J 10:29-34

Demirci F, Muştu M, Kaydan MB, Ülgentürk S (2011) Effects of some fungicides on Isaria farinosa, and in vitro growth and infection rate on Planococcus citri. Phytoparasitica 39:353-360. https://doi.org/10.1007/s12600-011-0168-2

Donatti AC, Furlaneto-Maia L, Fungaro MHP, Furlaneto MC (2008) Production and regulation of cuticle-degrading proteases from Beauveria bassiana in the presence of Rhammatocerus schistocercoides cuticle. Curr Microbiol 56:256-260. https://doi.org/10.1007/s00284-007-9071-y

Donzelli BGG, Krasnoff SB (2016) Molecular genetics of secondary chemistry in Metarhizium Fungi. In: Lovett B, Leger RJS (eds) Advances in genetics, vol 94. Elsevier, Amsterdam, pp 365-436

Donzelli BGG, Krasnoff SB, Churchill ACL et al (2010) Identification of a hybrid PKS-NRPS required for the biosynthesis of NG-391 in Metarhizium robertsii. Curr Genet 56:151-162. https://doi.org/10.1007/s00294-010-0288-0

Dou F, Wang Z, Li G, Dun B (2019) Microbial transformation of flavonoids by Isaria fumosorosea ACCC 37814. Molecules 24:1028. https://doi.org/10.3390/molecules24061028

Duo-Chuan L (2006) Review of fungal chitinases. Mycopathologia 161:345-360. https://doi.org/10.1007/s11046006-0024-y

Dymarska M, Janeczko T, Kostrzewa-Susłow E (2018a) Biotransformations of flavones and an isoflavone (daidzein) in cultures of entomopathogenic filamentous fungi. Molecules 23(6):1356. https://doi.org/10.3390/ molecules 23061356

Dymarska M, Janeczko T, Kostrzewa-Susłow E (2018b) Glycosylation of 3-Hydroxyflavone, 3-Methoxyflavone, quercetin and baicalein in fungal cultures of the Genus Isaria. Molecules 23(10):pii: E2477. https://doi.org/10.3390/ molecules 23102477

Fan Y, Liu X, Keyhani NO et al (2017) Regulatory cascade and biological activity of Beauveria bassiana oosporein that limits bacterial growth after host death. Proc Natl Acad Sci USA 114:E1578-E1586. https://doi.org/10.1073/pnas. 1616543114

Farias CP, Alves GS, Oliveira DC et al (2019) A consortium of fungal isolates and biochar improved the phytoremediation potential of Jacaranda mimosifolia D. Don and reduced copper, manganese, and zinc leaching. J Soils Sediments. https://doi.org/10.1007/s11368-019-02414-3

Feng M, Liao Z, Han L et al (2014) Enhancement of microbial hydroxylation of 13-ethyl-gon-4-ene-3,17-dione by $M e$ tarhizium anisopliae using nano-liposome technique. J Ind Microbiol Biotechnol 41:619-627. https://doi.org/10. 1007/s10295-014-1414-7

Feng P, Shang Y, Cen K, Wang C (2015) Fungal biosynthesis of the bibenzoquinone oosporein to evade insect immunity. Proc Natl Acad Sci 112:11365-11370. https://doi.org/10. 1073/pnas. 1503200112

Fiedler Z, Sosnowska D (2017) Side effects of fungicides and insecticides on entomopathogenic fungi in vitro. J Plant Prot Res 57:355-360. https://doi.org/10.1515/jppr-20170048

Forlani L, Juárez MP, Lavarías S, Pedrini N (2014) Toxicological and biochemical response of the entomopathogenic fungus Beauveria bassiana after exposure to deltamethrin. Pest Manag Sci 70:751-756. https://doi.org/10.1002/ps. 3583

Gola D, Dey P, Bhattacharya A et al (2016) Multiple heavy metal removal using an entomopathogenic fungi Beauveria bassiana. Bioresour Technol 218:388-396. https://doi.org/ 10.1016/j.biortech.2016.06.096

Gola D, Malik A, Namburath M, Ahammad SZ (2018) Removal of industrial dyes and heavy metals by Beauveria bassiana: FTIR, SEM, TEM and AFM investigations with $\mathrm{Pb}$ (II). Environ Sci Pollut Res 25:20486-20496. https://doi.org/ 10.1007/s11356-017-0246-1

Gonzalez R, Nicolau F, Peeples T (2015) N-alkane Solventenhanced Biotransformation of Steroid DHEA by Beauveria bassiana as Biocatalyst. J Adv Biol Biotechnol 2:30-37. https://doi.org/10.9734/jabb/2015/13541

Gonzalez R, Nicolau F, Peeples TL (2017) Optimization of the $11 \alpha$-hydroxylation of steroid DHEA by solvent-adapted Beauveria bassiana. Biocatal Biotransformation 35:103-109. https://doi.org/10.1080/10242422.2017. 1289183

Greenfield BPJ, Lord AM, Dudley E, Butt TM (2014) Conidia of the insect pathogenic fungus, Metarhizium anisopliae, fail to adhere to mosquito larval cuticle. R Soc Open Sci 1(2):140193. https://doi.org/10.1098/rsos.140193

Haruma T, Yamaji K, Ogawa K et al (2019) Root-endophytic Chaetomium cupreum chemically enhances aluminium tolerance in Miscanthus sinensis via increasing the aluminium detoxicants, chlorogenic acid and oosporein. PLoS ONE 14:e0212644. https://doi.org/10.1371/journal.pone. 0212644

Heilos D, Röhrl C, Pirker C et al (2018) Altered membrane rigidity via enhanced endogenous cholesterol synthesis drives cancer cell resistance to destruxins. Oncotarget 9:25661-25680. https://doi.org/10.18632/oncotarget. 25432

Hirose E, Neves PMOJ, Zequi JAC et al (2001) Effect of biofertilizers and neem oil on the entomopathogenic fungi Beauveria bassiana (bals.) vuill. and Metarhizium anisopliae (Metsch.) sorok. Brazilian Arch Biol Technol 44:419-423. 89132001000400013

Huarte-Bonnet C, Juárez MP, Pedrini N (2015) Oxidative stress in entomopathogenic fungi grown on insect-like 
hydrocarbons. Curr Genet 61:289-297. https://doi.org/10. 1007/s00294-014-0452-z

Huarte-Bonnet C, Kumar S, Saparrat MCN et al (2018) Insights into Hydrocarbon Assimilation by Eurotialean and Hypocrealean Fungi: roles for CYP52 and CYP53 Clans of Cytochrome P450 Genes. Appl Biochem Biotechnol 184:1047-1060. https://doi.org/10.1007/s12010-0172608-z

Hussein K, Hassan S, Joo J (2011) Potential capacity of Beauveria bassiana and Metarhizium anisopliae in the biosorption of $\mathrm{Cd}^{2+}$ and $\mathrm{Pb}^{2+}$. J Gen Appl Microbiol 57(6):347-355

Jaber LR, Enkerli J (2017) Fungal entomopathogens as endophytes: can they promote plant growth? Biocontrol Sci Technol 27:28-41. https://doi.org/10.1080/09583157. 2016.1243227

Jaihan P, Sangdee K, Sangdee A (2016) Selection of entomopathogenic fungus for biological control of chili anthracnose disease caused by Colletotrichum spp. Eur J Plant Pathol 146:551-564. https://doi.org/10.1007/s10658016-0941-7

Jajić I, Dudaš T, Krstović S et al (2019) Emerging fusarium mycotoxins fusaproliferin, beauvericin, enniatins, and moniliformin in serbian maize. Toxins (Basel) 11:357. https://doi.org/10.3390/toxins11060357

Jin K, Peng G, Liu Y, Xia Y (2015) The acid trehalase, ATM1, contributes to the in vivo growth and virulence of the entomopathogenic fungus, Metarhizium acridum. Fungal Genet Biol 77:61-67. https://doi.org/10.1016/j.fgb.2015. 03.013

Keswani C, Singh HB, Hermosa R et al (2019) Antimicrobial secondary metabolites from agriculturally important fungi as next biocontrol agents. Appl Microbiol Biotechnol 103:9287-9303

Keyhani NO (2018) Lipid biology in fungal stress and virulence: entomopathogenic fungi. Fungal Biol 122:420-429. https://doi.org/10.1016/j.funbio.2017.07.003

Khan S, Guo L, Maimaiti Y et al (2012) Entomopathogenic fungi as microbial biocontrol agent. Mol Plant Breed. https://doi.org/10.5376/mpb.2012.03.0007

Kidd PS, Alvarez-Lopez V, Becerra-Castro C et al (2017) Chapter three-potential role of plant-associated bacteria in plant metal uptake and implications in phytotechnologies. Adv Bot Res 83:87-126

Klieber J, Reineke A (2016) The entomopathogen Beauveria bassiana has epiphytic and endophytic activity against the tomato leaf miner Tuta absoluta. J Appl Entomol 140:580-589. https://doi.org/10.1111/jen.12287

Kouassi M, Coderre D, Todorova SI (2003) Effects of the timing of applications on the incompatibility of three fungicides and one isolate of the entomopathogenic fungus Beauveria bassiana (Balsamo) Vuillemin (Deuteromycotina). J Appl Entomol 127:421-426

Kozłowska E, Dymarska M, Kostrzewa-Susłow E, Janeczko T (2019) Cascade biotransformation of estrogens by Isaria fumosorosea KCh J2. Sci Rep 9:1-8. https://doi.org/10. 1038/s41598-019-47225-1

Kumar R, Kumawat N, Sahu YK (2017) Role of biofertilizers in agriculture. Pop Kheti 5:63-66

Lacey LA, Mercadier G (1998) The effect of selected allelochemicals on germination of conidia and blastospores and mycelial growth of the entomopathogenic fungus, Paecilomyces fumosoroseus (Deuteromycotina: Hyphomycetes). Mycopathologia 142:17-25. https://doi.org/10. 1023/A:1006963016316

Lacey LA, Grzywacz D, Shapiro-Ilan DI et al (2015) Insect pathogens as biological control agents: back to the future. J Invertebr Pathol 132:1-41. https://doi.org/10.1016/j.jip. 2015.07.009

Latteur G, Jansen JP (2002) Effects of 20 fungicides on the infectivity of conidia of the aphid entomopathogenic fungus Erynia neoaphidis. Biocontrol 47:435-444. https://doi. org/10.1023/A: 1015639017666

Liu BL, Tzeng YM (2012) Development and applications of destruxins: a review. Biotechnol Adv 30:1242-1254. https://doi.org/10.1016/j.biotechadv.2011.10.006

Lovett B, Leger RJS (2017) The insect pathogens. Microbiol Spectr. https://doi.org/10.1128/microbiolspec.funk-00012016

Mascarin GM, Jaronski ST (2016) The production and uses of Beauveria bassiana as a microbial insecticide. World $\mathbf{J}$ Microbiol Biotechnol 32:1-26. https://doi.org/10.1007/ s11274-016-2131-3

Mc Namara L, Dolan SK, Walsh JMD et al (2019) Oosporein, an abundant metabolite in Beauveria caledonica, with a feedback induction mechanism and a role in insect virulence. Fungal Biol 123:601-610. https://doi.org/10.1016/j. funbio.2019.01.004

McKinnon AC, Saari S, Moran-Diez ME et al (2017) Beauveria bassiana as an endophyte: a critical review on associated methodology and biocontrol potential. Biocontrol 62:1-17. https://doi.org/10.1007/s10526-016-9769-5

Meyling NV, Arthur S, Pedersen KE et al (2018) Implications of sequence and timing of exposure for synergy between the pyrethroid insecticide alpha-cypermethrin and the entomopathogenic fungus Beauveria bassiana. Pest Manag Sci 74:2488-2495. https://doi.org/10.1002/ps.4926

Mondal S, Baksi S, Koris A, Vatai G (2016) Journey of enzymes in entomopathogenic fungi. Pacific Sci Rev A Nat Sci Eng 18:85-99. https://doi.org/10.1016/j.psra.2016.10.001

Moonjely SS, Barelli L, Bidochka MJ (2016) Insect pathogenic fungi as endophytes. In: Lovett B, Leger RJS (eds) Advances in genetics. Elsevier, Amsterdam, pp 107-135

Neves PMOJ, Hirose E, Tchujo PT, Moino A (2001) Compatibility of entomopathogenic fungi with neonicotinoid insecticides. Neotrop Entomol 30:263-268. https://doi.org/ 10.1590/s1519-566x2001000200009

Nowak M, Soboń A, Litwin A, Różalska S (2019) 4-nnonylphenol degradation by the genus Metarhizium with cytochrome P450 involvement. Chemosphere 220:324-334. https://doi.org/10.1016/j.chemosphere. 2018.12.114

Olleik H, Nicoletti C, Lafond M et al (2019) Comparative structure-activity analysis of the antimicrobial activity, cytotoxicity, and mechanism of action of the fungal cyclohexadepsipeptides enniatins and beauvericin. Toxins (Basel) 11:514. https://doi.org/10.3390/toxins11090514

Pedrini N, Rosana C, Patricia Juarez M (2007) Biochemistry of insect epicuticule degradation by entomopathogenic fungi. Comp Biochem Physiol 146:124-137

Ramakuwela T, Hatting J, Bock C et al (2020) Establishment of Beauveria bassiana as a fungal endophyte in pecan (Carya 
illinoinensis) seedlings and its virulence against pecan insect pests. Biol Control 140:104102. https://doi.org/10. 1016/j.biocontrol.2019.104102

Ramesha A, Venkataramana M, Nirmaladevi D et al (2015) Cytotoxic effects of oosporein isolated from endophytic fungus Cochliobolus kusanoi. Front Microbiol 6:870. https://doi.org/10.3389/fmicb.2015.00870

Reddy DS, Reddy M, Pushpalatha M (2018) Interaction of fungicides with bio-control agents. J Entomol Zool Stud. 3:2098-2104

Ríos-Moreno A, Garrido-Jurado I, Resquín-Romero G et al (2016) Destruxin A production by Metarhizium brunneum strains during transient endophytic colonisation of Solanum tuberosum. Biocontrol Sci Technol 26:1574-1585. https://doi.org/10.1080/09583157.2016.1223274

Różalska S, Pawłowska J, Wrzosek M et al (2013) Utilization of 4-n-nonylphenol by Metarhizium sp. isolates. Acta Biochim Pol 60:677-682

Różalska S, Glińska S, Długoński J (2014) Metarhizium robertsii morphological flexibility during nonylphenol removal. Int Biodeterior Biodegrad 95:285-293. https:// doi.org/10.1016/j.ibiod.2014.08.002

Różalska S, Bernat P, Michnicki P, Długoński J (2015) Fungal transformation of $17 \alpha$-ethinylestradiol in the presence ofvarious concentrations of sodium chloride. Int Biodeterior Biodegrad 103:77-84. https://doi.org/10.1016/j.ibiod. 2015.04.016

Różalska S, Soliwoda K, Długoński J (2016) Synthesis of silver nanoparticles from Metarhizium robertsii waste biomass extract after nonylphenol degradation, and their antimicrobial and catalytic potential. RSC Adv 6:21475-21485. https://doi.org/10.1039/c5ra24335a

Różalska B, Sadowska B, Budzyńska A et al (2018) Biogenic nanosilver synthesized in Metarhizium robertsii waste mycelium extract-as a modulator of Candida albicans morphogenesis, membrane lipidome and biofilm. PLoS ONE 13:1-21. https://doi.org/10.1371/journal.pone. 0194254

Santi L, Beys da Silva WO, Berger M et al (2010) Conidial surface proteins of Metarhizium anisopliae: source of activities related with toxic effects, host penetration and pathogenesis. Toxicon 55:874-880. https://doi.org/10. 1016/j.toxicon.2009.12.012

Shah FA, Wang CS, Butt TM (2005) Nutrition influences growth and virulence of the insect-pathogenic fungus Metarhizium anisopliae. FEMS Microbiol Lett 251:259-266. https://doi.org/10.1016/j.femsle.2005.08. 010

Shah FA, Ansari MA, Watkins J et al (2009) Influence of commercial fungicides on the germination, growth and virulence of four species of entomopathogenic fungi. Biocontrol Sci Technol 19:743-753. https://doi.org/10. 1080/09583150903100807

Siewiera P, Różalska S, Bernat P (2017a) Efficient dibutyltin (DBT) elimination by the microscopic fungus Metarhizium robertsii under conditions of intensive aeration and ascorbic acid supplementation. Environ Sci Pollut Res 24:12118-12127. https://doi.org/10.1007/s11356-0178764-4

Siewiera P, Różalska S, Bernat P (2017b) Estrogen-mediated protection of the organotin-degrading strain Metarhizium robertsii against oxidative stress promoted by monobutyltin. Chemosphere 185:96-104. https://doi.org/10. 1016/j.chemosphere.2017.06.130

Silva WOB, Santi L, Berger M et al (2009) Characterization of a spore surface lipase from the biocontrol agent Metarhizium anisopliae. Process Biochem 44:829-834. https://doi.org/ 10.1016/j.procbio.2009.03.019

Sivakumar T, Jiji T, Naseema A (2019) Effect of pesticides used in banana agro-system on entomopathogenic fungus, $\mathrm{Me}$ tarhizium majus Bisch, Rehner and Humber. Int J Trop Insect Sci. https://doi.org/10.1007/s42690-019-00080-z

Skinner M, Parker BL, Kim JS (2014) Role of entomopathogenic fungi. In: Abrol DP (ed) Integrated pest management. Academic Press, Cambridge, pp 169-191

Sohrabi F, Jamali F, Morammazi S et al (2019) Evaluation of the compatibility of entomopathogenic fungi and two botanical insecticides tondexir and palizin for controlling Galleria mellonella L. (Lepidoptera: Pyralidae). Crop Prot 117:20-25

Sordon S, Popłónski J, Tronina T, Huszcza E (2017) Microbial glycosylation of daidzein, genistein and biochanin a: two new glucosides of biochanin A. Molecules 22(1):pii: E81. https://doi.org/10.3390/molecules22010081

Sotelo-Mundo RR, Lopez-Zavala AA, Garcia-Orozco KD et al (2007) The lysozyme from insect (Manduca sexta) is a cold-adapted enzyme. Protein Pept Lett 14:774-778

Stolarek P, Różalska S, Bernat P (2019) Lipidomic adaptations of the Metarhizium robertsii strain in response to the presence of butyltin compounds. Biochim Biophys ActaBiomembr 1861:316-326. https://doi.org/10.1016/j. bbamem.2018.06.007

Subbanna ARNS, Stanley J, Venkateswarlu V et al (2019) Toxicological prospects on joint action of microbial insecticides and chemical pesticides. In: Khan MA, Ahmad $\mathrm{W}$ (eds) Microbes for sustainable insect pest management. Springer, Cham, pp 317-340. https://doi.org/10.1007/9783-030-23045-6_12

Supakdamrongkul P, Bhumiratana A, Chanpen W (2010) Characterization of an extracellular lipase from the biocontrol fungus, Nomuraea rileyi MJ, and its toxicity toward Spodoptera litura. J Invertebr Pathol 105:228-235

Szewczyk R, Kuśmierska A, Bernat P (2018) Ametryn removal by Metarhizium brunneum: biodegradation pathway proposal and metabolic background revealed. Chemosphere 190:174-183. https://doi.org/10.1016/j.chemosphere. 2017.10.011

Taibon J, Sturm S, Seger C et al (2015) Quantitative assessment of destruxins from strawberry and maize in the lower parts per billion range: combination of a QuEChERS-based extraction protocol with a fast and selective UHPLCQTOF-MS assay. J Agric Food Chem 63:5707-5713. https://doi.org/10.1021/acs.jafc.5b01562

Tigano-Milani MS, Carneiro RG, De Faria MR et al (1995) Isozyme Characterization and Pathogenicity of Paecilomyces fumosoroseus and P. lilacinus to Diabrotica speciosa (Cleoptera: Chrysomelidae) and Meloidogyne javanica (Nematoda: Tylenchidae). Biol Control $5: 378-382$

Tkaczuk C, Harasimiuk M, Król A, Beres PK (2015) The effect of selected pesticides on the growth of entomopathogenic 
fungi Hirsutella nodulosa and Beauveria bassiana. J Ecol Eng 16:177-183. https://doi.org/10.12911/22998993/2952

Tkaczuk C, Majchrowska-Safaryan A, Panasiuk T, Tipping C (2019) Effect of selected heavy metal ions on the growth of entomopathogenic fungi from the Genus Isaria. Appl Ecol Environ Res 17:2571-2582. https://doi.org/10.15666/aeer/ 1702_25712582

Todorova SI, Coderre D, Duchesne RM, Côté JC (1998) Compatibility of Beauveria bassiana with selected fungicides and herbicides. Environ Entomol 27:427-433. https://doi. org/10.1093/ee/27.2.427

Urbaniak M, Stępień Ł, Uhlig S (2019) Evidence for naturally produced beauvericins containing $N$-methyl-tyrosine in Hypocreales fungi. Toxins (Basel) 11:182. https://doi.org/ $10.3390 /$ toxins 11030182

Vega FE (2018) The use of fungal entomopathogens as endophytes in biological control: a review. Mycologia 110:4-30. https://doi.org/10.1080/00275514.2017. 1418578

Wang C, Leger RJS (2007) The MAD1 adhesin of Metarhizium anisopliae links adhesion with blastospore production and virulence to insects, and the MAD2 adhesin enables attachment to plants. Eukaryot Cell 6:808-816. https://doi. org/10.1128/EC.00409-06

Wang Q, Xu L (2012) Beauvericin, a bioactive compound produced by fungi: a short review. Molecules 17:2367-2377. https://doi.org/10.3390/ molecules 17032367

Wang B, Kang Q, Lu Y et al (2012) Unveiling the biosynthetic puzzle of destruxins in Metarhizium species. Proc Natl Acad Sci 109:1287-1292. https://doi.org/10.1073/pnas. 1115983109

Wang H, He Z, Luo L et al (2018a) An aldo-keto reductase, Bbakr1, is involved in stress response and detoxification of heavy metal chromium but not required for virulence in the insect fungal pathogen, Beauveria bassiana. Fungal Genet Biol 111:7-15. https://doi.org/10.1016/j.fgb.2018.01.001

Wang X, Gong X, Li P et al (2018b) Structural diversity and biological activities of cyclic depsipeptides from fungi. Molecules 23:169. https://doi.org/10.3390/ molecules 23010169

Wang X, Xu J, Wang X et al (2019a) Isaria fumosorosea-based zero-valent iron nanoparticles affect the growth and survival of sweet potato whitefly, Bemisia tabaci (Gennadius). Pest Manag Sci 75:2174-2181

Wang Z, Chen Z, Jiang Z et al (2019b) Cordycepin prevents radiation ulcer by inhibiting cell senescence via NRF2 and AMPK in rodents. Nat Commun 10:2538. https://doi.org/ 10.1038/s41467-019-10386-8

Weng Q, Zhang X, Chen W, Hu Q (2019) Secondary metabolites and the risks of Isaria fumosorosea and Isaria farinosa. Molecules 24(4):pii: E664. https://doi.org/10. 3390/molecules24040664

Wu S, Kostromytska OS, Goble T et al (2020) Compatibility of a microsclerotial granular formulation of the entomopathogenic fungus Metarhizium brunneum with fungicides. Biocontrol 9:113-123. https://doi.org/10.1007/ s10526-019-09983-9

Xia Y, Luo F, Shang Y et al (2017) Fungal cordycepin biosynthesis is coupled with the production of the safeguard molecule pentostatin. Cell Chem Biol 24:1479-1489.e4. https://doi.org/10.1016/j.chembiol. 2017.09.001

Xiong Z, Wei Q, Chen $\mathrm{H}$ et al (2006) Microbial transformation of androst-4-ene-3,17-dione by Beauveria bassiana. Steroids 71:979-983. https://doi.org/10.1016/j.steroids.2006. 07.007

$\mathrm{Xu}$ Y, Orozco R, Wijeratne EMK et al (2009) Biosynthesis of the cyclooligomer depsipeptide bassianolide, an insecticidal virulence factor of Beauveria bassiana. Fungal Genet Biol 46:353-364. https://doi.org/10.1016/j.fgb.2009.03. 001

Yang B, Liu H, Yang J et al (2018) New insights on bioactivities and biosynthesis of flavonoid glycosides. Trends Food Sci Technol 79:116-124. https://doi.org/10.1016/j.tifs.2018. 07.006

Yosri M, Abdel-Aziz MM, Sayed RM (2018) Larvicidal potential of irradiated myco-insecticide from Metarhizium anisopliae and larvicidal synergistic effect with its mycosynthesized titanium nanoparticles (TiNPs). J Radiat Res Appl Sci 11:328-334. https://doi.org/10.1016/j.jrras. 2018.06.001

Zhang YJ, Liu X, Wang M (2008) Clonig, expression and characterization of two novel cuticle-degrading serine proteases from the entomopathogenic fungus Cordyceps sinensis. Res Microbiol 159:462-469

Zhao H, Lovett B, Fang W (2016) Genetically engineering entomopathogenic fungi. In: Lovett B, Leger RJT (eds) Advances in genetics, vol 94. Elsevier, Amsterdam, pp 137-163

Zimmermann G (2008) The entomopathogenic fungi Isaria farinosa (formerly Paecilomyces farinosus) and the Isaria fumosorosea species complex (formerly Paecilomyces fumosoroseus): biology, ecology and use in biological control. Biocontrol Sci Technol 18:865-901. https://doi. org/10.1080/09583150802471812

Publisher's Note Springer Nature remains neutral with regard to jurisdictional claims in published maps and institutional affiliations. 